Meta

Journal des tradlucteurs

Translators' Journal

\title{
L'art de traduire selon Maximillian Volochine : la fidélité et la créativité — « Le Cygne " de S. Mallarmé
}

\section{Vera Adamantova}

Volume 36, numéro 2-3, juin 1991

URI : https://id.erudit.org/iderudit/001853ar

DOI : https://doi.org/10.7202/001853ar

Aller au sommaire du numéro

Éditeur(s)

Les Presses de l'Université de Montréal

ISSN

0026-0452 (imprimé)

1492-1421 (numérique)

Découvrir la revue

Citer cet article

Adamantova, V. (1991). L'art de traduire selon Maximillian Volochine : la fidélité et la créativité — « Le Cygne » de S. Mallarmé. Meta, 36(2-3), 461-470. https://doi.org/10.7202/001853ar d'utilisation que vous pouvez consulter en ligne. 


\section{L'ART DE TRADUIRE SELON MAXIMILLIAN VOLOCHINE: LA FIDÉLITÉ ET LA CRÉATIVITÉ - «LE CYGNE» DE S. MALLARMÉ}

VERA ADAMANTOVA

University of Western Ontario, London, Ontario, Canada

Le sonnet de Stéphane Mallarmé «Le Cygne», écrit en 1864-1865, a paru dans la Revue indépendante en mars $1885^{1}$. En Russie, il a été traduit pour la première fois par Maximilian Volochine à la demande d'une riche Russe qui a tenu un salon littéraire à Paris, Alexandra Bauler (Holshtein), pour son article «Stéphane Mallarmé» publié à Saint-Petersburg en 1905 dans la revue les Questions de vie (Voprosy zhizni) ${ }^{2}$. Plus tard, Val. Brussov et In. Annenski l'ont traduit aussi respectivement en 1907 et $1908^{3}$. La traduction d'Annenski représente une «libre» interprétation sur le thème donné; la traduction de Brussov, au contraire, transmet presque mot à mot le thème et les images, mais ignore totalement le ton et l'esprit du style mallarméen. Volochine seul a su saisir «la jouissance du poème» (Mallarmé) - sa suggestion - en utilisant la méthode de «l'équivalence créative» dont nous allons parler.

La traduction de Volochine a attiré immédiatement l'attention non seulement du public russe, mais aussi du public français. René Gil, un des disciples proches de Mallarmé, fondateur de «la théorie d'instrumentation du vers», écrit au sujet de l'article de Bauler:

... Dans cet article réfléchi, formidable par sa compréhension intuitive de Mallarmé, nous trouvons des parfaites traductions du «Miroir» et du «Cygne» faites en vers par Mr. Maximillian Volochine qui a transmis avec toute précision non seulement le texte de l'original mais aussi $l^{\prime}$ esprit de création de Mallarmé ${ }^{4}$ (italiques = V.A.).

Ces derniers mots saisissent l'essentiel dans la méthode de traduction par Volochine, c'est la reproduction précise du texte avec l'esprit de création de l'original.

\section{L'AFFINITÉ DE DEUX POÈTES : L'EXIGENCE PRÉALABLE DE BONNE TRADUCTION}

Le sonnet de Mallarmé «Le Cygne» passe pour un de ses chefs-d'œuvre. En effet, en lui il a exprimé les traits fondamentaux de son esthétique - la perfection de forme, «le don de suggestion et l'art d'analogie» (Ghil) ${ }^{5}$, la sonorité et l'harmonie de vers. Traduire ce sonnet en russe représente une difficulté considérable non seulement sur le plan du contenu, des images et des symboles, mais aussi au point de vue de la structure, de la forme, de la syntaxe et des sons, à tel point que cette œuvre est saturée, raffinée et perfectionnée. Il faut remarquer que la poésie de Mallarmé n'était pas très souvent traduite en russe, même à l'époque du symbolisme, quoique, au contraire, sa théorie du symbole était bien connue et étudiée en Russie au début du siècle. L'explication se trouve dans le raffinement et la subtilité du vers mallarméen. Ainsi Mallarmé appartient à ce groupe d'auteurs qui était qualifié par Volochine comme «difficile, presque intradui-

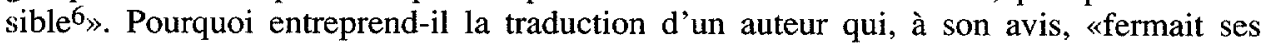
jdées dans les cornues alchimiques, dans les cristaux magiques et dans les formules 
algébräques $7_{\gg}$ ? Il faut chercher la réponse dans la personnalité de Volochine et dans sa philosophie de l'art et de la création. Comme Gothe, il croyait que la discipline et la difficulté de création, ainsi que la restriction en art étaient nécessaires. Dans la lettre à sa mère il écrivait :

... je fais dans l'art seulement ce qui est difficile... les traductions - je choisis des auteurs très difficiles, presque intraduisibles... ${ }^{8}$ (italiques = V.A.)

Autre constatation - «Le Cygne» est écrit en forme de sonnet, la forme rigide qui impose une certaine restriction pour la création comme telle. Volochine lui-même produit 66 sonnets en russe et traduit 8 sonnets des poètes français ${ }^{9}$. Il est connu que le sonnet, la forme typiquement romaine, $n$ 'a pas pu s'établir en Russie à cause de son exclusivité. Non sans raison, Valéry Brussov reconnaît:

... Il se peut que personne n'ait le sonnet correct, sauf moi-même et Max Volochine ${ }^{10} \ldots$

En ce qui concerne les idées de Volochine sur l'art en général et la poésie en particulier, il y a la réminiscence de celles de Mallarmé. Pour Volochine, «l'art est le point de diamant entre l'être et ce qui est hors de l'être», la poésie est «l'apprentissage majestueux

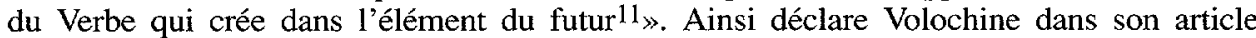
«Horomedon» publié en 1909, mais écrit bien avant, dans les années 1904-1905 quand l'influence du symbolisme français en Russie était considérable. Dans cet article il s'engage dans un programme d'unification des arts plastiques, de la musique et de la poésie. Le signifié du Verbe devient primordial ainsi compris comme «un être vivant», comme «une force incantée», comme «un but et non un moyen ${ }^{12}$ ». Ceci est parce que, - écrit-t-il - ,

... Tout avant de commencer à exister comme la volonté, la forme, le masque était le Verbe, et il n'y a rien autour de nous que ne renfermerait pas le Verbe caché dans la substance sombre: et le son, et l'odeur, et la couleur - toutes ces perceptions externes ne sont que les brûlures du Verbe ardent étant sous l'emprise des masques de l'Univers, du Verbe qui donne sa voix du plus profond de la prison vers le poète et attend de lui son nom, son évasion ${ }^{13} \ldots$

Cette aspiration à la perfection, à la beauté, à l'idéal réunissent Mallarmé et Volochine en ce qui concerne la poésie. Leur idéal était la conscience du style, ce qui devient pour Mallarmé «le blanc souci de notre toile ${ }^{14}$ et pour Volochine :

Pour le métier et l'esprit la seule voie est la restriction de soi-même ${ }^{15}$.

Ainsi nous comprenons pourquoi Volochine voulait reproduire Mallarmé «intraduisible», hermétique dans sa langue de création. Ici se trouve la première règle de jeu c'est de traduire le poète proche de sa conception de l'art et de son style individuel.

\section{LA MÉTHODE : LA CO-CRÉATION (SOPRICHASTIE)}

Volochine n'était pas un traducteur professionnel dans le sens contemporain du mot. La traduction pour lui faisait parti du métier du poète, de critique et d'artiste. Il traduisit toute sa vie (1877-1932) car il considérait la traduction comme une partie intégrale du processus littéraire, de l'école esthétique. Il était partisan d'un syncrétisme culturel en croyant que la traduction littéraire apporterait une interinfluence de toutes les littératures du monde et que tous les arts du monde allaient se rapprocher en formant un ensemble. C'est pourquoi, pour lui, la traduction littéraire est mise sur le même niveau que la création originale. Il écrivait dans son article «Émile Verhaeren et Valéry Brussov» que la traduction poétique représente la coopération (coparticipation) à la création ou «la cocréation» (soprichastie) ${ }^{16}$ du poète et du traducteur car «la traduction est destinée... à remplacer l'original en russe ${ }^{17} \gg$. Une bonne traduction est possible - affirme Volochine — s'il s'agit d'une communion à l'acte de création elle-même : 
Si cette communion existe, la merveille d'art est accomplie. On ne peut pas traduire un seul vers, mais on peut recréer beaucoup de vers dans sa langue maternelle. La communion à l'acte de création donne les droits grands et absolus sur le vers traduit car le poète-cocréateur dispose les trésors de l'autre langue et en trouve les images et les mots loin d'être identiques, mais égaux en force, équivalents ${ }^{18}$ (italiques $=$ V.A.).

Par conséquent, la définition de la traduction comme «la cocréation» (soprichastie) avec «la force égale d'expression» est liée à la définition de la traduction exacte ou de la fidélité. La traduction littérale ne garantit pas la fidélité à l'original parce que la traduction fidèle de l'œuvre poétique au sens strict du mot est impossible, selon Volochine:

... dans la traduction poétique il est nécessaire de sacrifier des éléments qui ne sont pas si importants pour la chose principale: si le vers est fait sur les jeux des sons, le traducteur renonce inévitablement au sens précis et, principalement, à la syntaxe, en essayant de trouver un effet des sons à force égale. Si, donc, le principal est dans le contenu, dans l'image, dans le développement syntaxique de la phrase, le traducteur doit sacrifier partiellement la rime ${ }^{19}$ (italiques = V.A.)

La haute appréciation d'équivalence pour Volochine était la reproduction de la voix du poète. Il a répété les paroles de T. Gauthier qui a dit qu'avec la mort du poète avant tout meurt sa voix, et la reproduction artistique du poète mort est «une des tâches les plus difficiles de la littérature ${ }^{20} \gg$. La voix lyrique intime du poète, son aisance et sa simplicité d.expression, sa pureté et sa légèreté de style ne peuvent être reproduites dans l'autre langue que par un poète dont la méthode de création a de l'affinité avec le poète traduit. Comme exemple de la haute équivalence «de voix» Volochine cite F. Sologub:

... Il a pu réaliser ce qui semblait impossible et inconcevable: c'est de reproduire dans le vers russe la voix de Verlaine ${ }^{21} \ldots$

Plus loin, Sologub comme traducteur est apprécié de la façon suivante :

... C'est si semblable à Verlaine... Verlaine lui-même a commencé à parler en vers russe... Verlaine devient le poète russe ${ }^{22} \ldots$ (italiques $=$ V.A.)

Transmettre la voix intime d'un poète étranger pour Volochine ne se faisait pas seulement dans la reproduction fidèle du contenu, des rythmes et des rimes séparément, dans leur signification absolue, mais dans la juste corrélation entre des sons et leurs combinaisons d'une part, et le sens des mots, d'autre part, c'est-à-dire en liant le sens avec le son en reproduisant l'intonation individuelle. Rappelons que pour Roman Jakobson, l'intraduisibilité de la poésie se trouve dans l'impossibilité de transmettre la substance de la langue poétique, sa paronomase ${ }^{23}, c^{\prime}$ est-à-dire une structure phono-sémantique du poème dans laquelle le son et le sens correspondent. Léon Robel détermine la reproduction de la voix du poète de la façon suivante :

... l'opération par laquelle on re-produit ce que nous avons appelé la structure profonde, phono-sémantique du poème... C'est cela sans doute que les poètes traducteurs saisissent intuitivement lorsqu'ils parviennent à restituer ce qu'ils appellent le «ton», la «voix»du poète original ${ }^{24}$.

En comparant cette définition avec celle de Volochine, on peut seulement admirer la profondeur de sa pensée qui n'a pas perdu sa signification à présent dans la théorie et la pratique de la traduction. Malheureusement, Volochine n'a pas été apprécié à sa juste valeur, mais cela ne veut pas dire qu'il a été oublié définitivement dans son pays. V. Perelmouter écrit que dans un questionnaire fait en URSS dans les années 1980 sur la question de savoir quelles sont les meilleures traductions poétiques du $20^{\mathrm{e}}$ siècle, parmi les dix meilleurs étaient deux traductions de Volochine : «Au Nord» et «Michelangelo» 
de Verhaeren. À l'ouest, l'art de traduire selon Volochine est bien exprimé par Emmanuel Rice :

... dans la plupart de ses [Volochine] traductions étonne son habileté qui ne sacrifie ni l'image, ni la rime, et qui suit presque littéralement les mots de l'original, qui garde le style, les traits individuels de l'auteur traduit et beaucoup d'autres éléments théoriquement imperceptibles. S'il renonce parfois à quelque chose, ce ne sont qu'en réalité à des aspects secondaires, faibles de l'original ${ }^{25}$.

\section{ANALYSE DE L'IMAGERIE DE TRADUCTION DU «LE CYGNE» DE MALLARMÉ}

Mon analyse comparative des deux poètes suivra cette méthode dont nous avons parlé plus haut, «la méthode à force égale» (ravnosilie) ou «la cocréation» (soprichastie) par laquelle Volochine a réussi à transmettre des images, des structures et des sons mallarméens pour rendre en poème la même impression qu'en français, sans que les idées de l'original en souffrent. Puisqu'il n'est pas possible d'analyser chaque niveau de stylistique en détail dans cette communication, je me limiterai à examiner les techniques d'imagerie, tout en recommandant ma thèse de doctorat pour une plus ample analyse du «Cygne». Pour le bref compte rendu de l'analyse, je vous renvoie au tableau sommaire.

Le thème général du poème - le resplendissement éternel du génie, la fidélité à la Muse et la lutte inutile de chaque jour contre l'impossibilité de poète à atteindre l'idéal - est transmis par Volochine d'une manière adéquate. Mais la version russe prend la forme plus concrète: la pensée abstraite de Mallarmé acquiert la signification visible de la lutte inutile du poète-Cygne dans son aspiration vers l'idéal - «le pays pour s'envoler» («strany, chtob ouletet $\left.{ }^{\prime 7}\right)$ ), où le poète s'adonne entièrement à sa Muse. Examinons ces images textuellement.

Dans le premier quatrain, Mallarmé demande si le jour «nouveau, vierge (pur), énergique et beau» permettra au poète de s'envoler avec l'élan, de se lever grâce à son inspiration et de finir avec l'incertitude devant le papier, c'est-à-dire de finir tous ces poèmes que sont restés inachevés. «Le aujourd'hui» (1) est personnifié, il est employé avec l'article ce qui représente un archaïsme. Selon Chassé, c'est motivé: pour Mallarmé «le aujourd'hui» est égal à «la vie de poète», son présent ${ }^{26}$. Volochine «rétrécit» l'image du jour, de vie en général et parle de la vie du poète en remplaçant «le aujourd'hui» par le pronom «il» $(o n)^{27}$. Il s'agit d'un oiseau d'une Beauté extraordinaire qui représente le symbole «jour-vie». La version russe le rend par ses attributs - «bezoumiem kryla» (2) «avec l'aile délirante (aile ivre)», - «poletov skovannykh» (4) - «les vols entravés (paralysés)», — «v kraze izvivnykh linii» (1) — «dans la beauté des lignes tordues (le bel)».

Le deuxième quatrain russe dévoile l'énigme, il s'agit d'un oiseau-Cygne. «Cygne» avec la majuscule, c'est-à-dire le Poète en général. Chez Mallarmé, «un cygne» devient «le Cygne» seulement dans la conclusion - la chute du sonnet. Cela diminue la qualité énigmatique de la version russe, mais les deux symboles mallarméens (deux oiseaux) transmis par les attributs («puissant», «vierge», «dans la beauté des lignes tordu»...) ont un rapport avec la vie du Poète, la vie qui n'est pas possible sans cet état «d'ivresse», de «délire», «de folie». Donc, les macro-images sont identiques, les micro-images sont rétrécies, mais la liaison avec l'original est là. «Les vols qui n'ont pas fui» (4) «poletov scovannykh» (4) — «les vols entravés, paralysés» sont des poèmes magnifiques qui sont restés dans la conscience du poète, les poèmes non écrits sur le papier. Chez Volochine, cette impossibilité de les écrire est exprimée par «volnogo teer nevolit dal'» (10), ce qui représente l'impuissance poétique dont nous parlerons plus loin. Littéralement «les vols qui ne sont pas fui» doivent être traduits comme «les vols qui ne se sont pas envolés»; «Skovannykh» («paralysés») se trouve lié avec le symbole de base de Mallarmé, le symbole de «l'espace gelé», «ka glace». Mais «scovannykh» (paralysés ou 
entravés) suggère «pris par la glace». Volochine transmet par la connotation l'image de la glace qui représente la conscience du Poète quand il se souvient du rêve perdu, la Beauté.

Le premier symbole de l'oiseau comme «jour = vie» est suivi par l'image du cleuxième oiseau — «Lebed" prejnikh dnei» (5), «Un Cygne d'autrefois» (5). Le poète se souvient du temps passé quand il pouvait écrire les poèmes mais il a perdu le moment de l'inspiration. Ce symbole de l'oiseau = inspiration est transmis en russe par connotation en séries d'images suivantes:

(6) «ne vzvit'sia» - «ne pas se déployer»,

(6) «ne zapet'»- «ne pas chanter»,

(7) «ouletet'»-«s'envoler»,

(7) «v pesne»- «dans la chanson».

(7) «strany» — «du pays»

“La région où vivre» (7) - «strany, chtob ouletet'» (7) - «le pays pour s'envoler» est le thème principal de l'œuvre. Dans la version russe «strana» (pays) est équivalent de l'idéal de poésie-muse. Ici vient à l'esprit l'article de Volochine «Horomedon» où l'image de Mallarmé «le pays pour s'envoler» est employée comme un parallèle au «cristal du futur Univers $28 》$, une paraphrase pour la poésie, ce qui dans sa compréhension est la force incantée, la prophétie du futur. C'est pour cela qu'elle devient «le chant» (pésnia). Par conséquent, la vie d'un poète se trouve être la création de «chansons» (la poésie idéale). Donc «chanté la région où vivre» est compris et transmis fidèlement.

L'image de l'impuissance poétique ( «se délivrer ${ }^{29}$ )) est traduite par connotation: se livrer $=\langle o n$ znaet...»; il sait que son éclatement et sa révolte sont inutiles car la poésie idéale, «le pays» (strana), la poésie de conscience est impossible à cause de sa propre faiblesse poétique. Cette image de l'impuissance poétique se continue dans le premier tercet - «Dal'» (le lointain, l'espace) symbolise la matière ${ }^{30}$, c'est-à-dire la limitation de la conscience du poète qui crée la poésie idéale dans les entraves de laquelle il se trouve sans qu'il puisse se libérer de son propre «esclavage» - la création, car le poète se sent obliger de créer. Il est libre de ne pas écrire des poèmes mais il y a une force incantée qui le contraint - «nevolit». Selon J. Gengoux, pour Mallarmé «l'espace - la matière inflige au Cygne un supplice terrible ${ }^{31} \gg$. La traduction transmet cela car «l'espace contraint un libre» (10), ce qui signifie que la poésie est le supplice de l'âme et du corps, mais le poète n'a pas de force pour s'en libérer car elle (la poésie) vient du ciel. Une telle compréhension de la poésie par Volochine est confirmée par Mallarmé quand il écrivait que la poésie pour le poète est «la seule tâche spirituelle 32 ».

Enfin, le symbole d'oiseau-poète se continue dans le deuxième tercet. Le Cygne ne juge pas son entourage, son milieu vulgaire où il se trouve, il blâme sa propre impuissance devant le papier blanc, car il a laissé échapper le moment d'inspiration («bezoumiem kryla» - «avec l'aile délirante» (2), et il n'a pas écrit «la chanson» - «pesnia» (7), parce qu'il n'a pas utilisé l'énergie et l'inspiration d'autrefois :

«On cheei otriaxnet smertel' no bessil' e» - «il secouera avec son cou l'impuissance mortelle» (9), et il n'aura pas assez d'énergie pour faire revenir le paradis perdu «ozero metchty» - «le lac de rêve» (3). Le résultat, c'est la poésie elle-même («dal»«l'espace», 10) et non «ne pozor zemli, chto primorozil kryl'ia» (11) - «une honte du sol qui a soudé les ailes par la gelée». Ici «pozor» («honte, infamie, déshonneur») ne traduit pas «l'horreur» (11), mais sont à force égale par leur sémantique. Dans la première version (1905), «l'horreur» était traduite par «kochmar» (le cauchemar), puis Volochine l'a corrigée en «pozor», ce qui est bien, car cela transmet la connotation phono-sémantique de «l'horreur du sol (avec deux sons «o»). Remarquons que «pozor» en russe ancien veut dire «zrélichche» (le spectacle). Le poète se punit lui-même et s'immobilise au songe froid du mépris — «i stynet $v$ gordykh snakh» (13) - «s'immobilise dans les rêves 
hautains (orgueilleux)». C'est vrai, il y a une certaine pose dans tout cela, alors «pozor = zrelichche» (honte $=$ spectacle) fait écho au «mépris» (13), c'est une parfaite adaptation de l'image mallarméenne.

De la même façon, «magnifique» (6) n'est pas traduit littéralement, sa connotation est là dans «poryve gordoi mouki» (5) - «dans l'élan d'une souffrance orgueilleuse». Les images d'un oiseau «magnifique» et «orgueilleux» sont juxtaposées. Le traducteur trouve l'analogie d'images - «gordykh snakh» («les rêves hautains») - «nadmennouiu pechal'» («la tristesse arrogante» - «nenoujnogo izgnania» («l'exil inutile»). Notons que «gordykh snakh» («les rêves de mépris, 13), la sémantique de «gordyi» («hautain, orgueilleux») en russe s'approche de la signification des mots «froid» («xolodnyi») «hautain (orgueilleux)» — «gordyi» («froid»).

En ce qui concerne «nadmennouiu pechal'» («la tristesse arrogante»), elle représente l'adaptation de l'image «mépris». Dans l'original, il n'y a pas de «tristesse arrogante», mais tout le ton, l'esprit du sonnet est hautement pessimiste exprimant une «indéniable tristesse, résignée» (Chadwick) ${ }^{33}$.

Volochine est exact dans sa transposition du pessimisme mallarméen quand il finit le sonnet par «okouitannyi v nadmennouiu pechal» (14) - «enveloppé dans la tristesse arrogante», ce qui représente la chute du sonnet et en même temps se trouve en liaison avec le premier quatrain : l'impossibilité d'écrire les beaux vers - «les vols entravés» (4) le met au désespoir ( «pechal», 14).

Le deuxième symbole de Mallarmé, le symbole de la transparence de la conscience, du Néant, de la pureté absolue est exprimé par les images de l'espace gelé: «lac dur oublié», «sous le givre», «le transparent glacier», «du stérile hiver», «l'horreur du sol où le plumage est pris», «blanche agonie». «Ce lac dur oublié» est traduit par analogie «ozero mechty» («le lac du rêve»). Cela semble, au premier regard, être loin du texte. Mais la lecture attentive de «ce lac dur oublié que hante» (3) décèle la sémantique de l'image en traduction. «Hanter», outre son sens direct, signifie aussi en russe «obséder = hanter», «obséder» par des rêves, des idées ${ }^{34}$. Le lac, comme la mer aussi, représente pour Mallarmé l'angoisse de la Beauté perdue ${ }^{35}$. Le poète se rappelle la Beauté («Beauté» dans le sens philosophique du mot) qu'il a perdue. (À ce propos, Mauron interprète le rêve comme la foi perdue ${ }^{36}$ ). Donc, «ozero mechty» «le lac du rêve») a une force égale dans la transposition de l'image mallarméenne.

En ce qui concerne «oublié», nous le trouvons adapté en «prozrachno-sinii led» («la glace bleue-transparente» (4). Pour Chassé qui utilise l'étymologie de Littré «oublié $=$ lividus», «oublié = ce lac bleuâtre» (3). Dans la version russe cela devient «ozero ... prozrachno-sinii led», le lac est devenu «la glace transparente bleue foncée»: «sinii» («bleue») et «prozrachny» («transparent») sont «accrochés»l'un à l'autre. Nous savons que le bleu foncé ne peut pas être transparent, mais un tel mélange des perceptions est dans le style de Mallarmé. Ici la couleur de la glace («la glace bleue») signifie les «rêves stériles, infructueux, vains». La couleur bleue chez Mallarmé signifiait par association l'image de la Beauté en dehors du temps; par contre, la couleur blanche suggérait l'idéal de pureté (rappelons: «le blanc souci de notre toile ${ }^{37}$ »). L'image de l'hiver avec ses attributs («sous le givre», «lac dur», etc.) représente pour Mallarmé l'ennui, le Néant, l'Inutile; autrement dit, c'est le refus de vivre = de créer. Pour la reproduction de cette image, Volochine emploie l'interversion des couleurs ou l'hypallage synesthétique (le terme de S. Johansen) utilisée couramment par les symbolistes russes aussi bien que par les symbolistes français. En russe existe «l'hiver blanc» et non «l'ennui blanc» (8). L'emploi de cet épithète abstrait par Volochine décèle la transcendance de l'image «zima $v$ sian'i beloi skouki» (8) («l'hiver dans le resplendissement de l'ennui blanc». Cela ajoute à la traduction une force de caractère impressionniste et suggestif, à savoir que 
Volochine a réussi à saisir l'irrationnel de l'expression et le manque de liaison logique entre les mots comme chez Mallarmé. Il y a un autre hypallage synesthétique dans l'original: $(9,10)$ «Tout son col secouera cette blanche agonie. Par l'espace infligée à l'oiseau qui le nie» où «l'espace blanc» est possible et non «blanche agonie». La traduction de cette interversion-métonymie est moins bien réussie que la précédente car «smertel'no bessil' e» («l'impuissance mortelle») (9) a un rapport avec l'image de «l'espace» («dal'») [la poésie = matière] qui inflige au poète [une] «indéniable tristesse résignée 38 ». En russe «la mort» est évoquée explicitement («smertel" nœ» — «mortelle»), en français elle est seulement suggérée. Nous sommes en présence de la technique de l'image «étendue» quand l'image similaire communique le sens plus fort. Une autre image semblable représente la traduction du «Fantôme». Le traducteur emploie la métonymie : les signes de l'objet - «Fantôme» sont présents («belizna» — «blancheur», «scovan» — «immobilisé», «odeanie» - «vêtements», «okoutannyi» - «enveloppé», 12). Ici l'image du «Fantôme» est «rétrécie» (pour l'image similaire le sens le plus faible est communiqué).

«La blancheur» («belizna») ne traduit pas «son pur éclat» (12), l'image est saisie par l'analogie. Pour Mallarmé, la page blanche est équivalente au poème inachevé parfait parce qu'il existe dans sa conscience.

Le lexique employé par le traducteur pour déceler les images est lié à l'esthétique de Mallarmé et surtout au lexique traditionnel spécifique des symbolistes russes. Il s'agit de la tendance à l'abstraction, de la perte de la concrétisation, de l'impressionnisme des perceptions, de l'emploi des archaïsmes et des négativismes ${ }^{39}$.

Il est naturel que la langue de traduction suive cette tradition. Cependant, ce qui constitue le mérite de la traduction, c'est que Volochine a saisi la poétique d'imagerie mallarméenne où la pensée directe est exclue, où une image s'attache à l'autre plutôt qu'à l'objet. L'humeur de suggestion est créée par la juxtaposition des mots-images dit motsclefs qui permettent de reproduire les images comme des relations et non comme des objets. Par exemple, deux symboles de Mallarmé sont exprimés par deux groupes de dénotats (signifiés):

1) l'oiseau - jour $=$ vie

2) la conscience $-=$ l'espace gelé

Les deux groupes de dénotats se trouvent en corrélation entre eux-mêmes et aussi avec le troisième groupe des mots-clefs, les connotats (signifiants) traduisant les attributs de la poésie-muse: «le lac de rêve», «le pays», «l'espace», «l'impuissance mortelle», «le tourment orgueilleux», «contraint». Le parallélisme des relations se présente comme ceci :

1) «poletov scovannykh» («vols entravés») - «nevolit» («contraint») - «smertel'nae bessil'e» («l'impuissance mortelle»);

2) «ne zapet'» («ne pas chanter») - «on scovan» («il s'immobilise») - «dal'» («l'espace»);

3) «bezoumiem kryla» («avec l'aile délirante») - «zemnogo odean'a» («des vêtements du sol») - «v gordykh snakh» («dans les rêves hautains»).

Donc, la tendance stylistique de l'imagerie de la traduction représente l'équivalence créative de celle de Mallarmé. Volochine a trouvé les techniques de transposition des images d'une langue à l'autre à l'aide de ses moyens à force égale (ravnosilie), ce que nous pouvons appeler «traduction de poésie du contexte». En somme, en employant la définition de Léon Robel, il a réussi à reproduire «une imitation d'une création et une création d'une imitation $40_{\gg,}$ ou comme disait Volochine, le traducteur doit:

Prendre l'œuvre à cœur, puis la faire naître de nouveau, sans quoi la traduction créative ne peut pas être 41 . 


\section{Le tableau-sommaire de la traduction du poème}

\section{«Le cygne» de Mallarmé par M. Volochine}

\section{Expression thématique}

Image identique

Image similaire-le sens plus faible (le rétrécissement)

Image similiare-le sens plus fort (l'étendue)

Image par connotation

Adaptation par l'association de l'image

Image par l'analogie

Non concordant

Lexique

Ton, voix

\section{Harmonie du vers}

Forme

Structure strophique

Nombre de lignes

Mètre

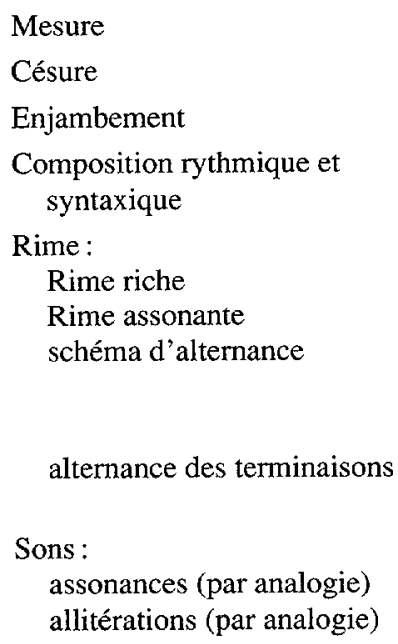

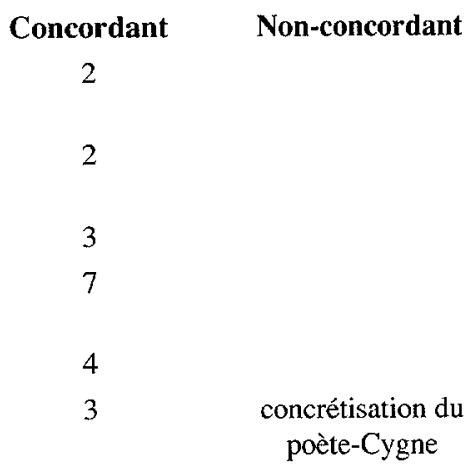

adéquate

adéquate

sonnet

$4: 4: 3: 3$

14

Analogue

d'alexandrin «iambe de 6 pieds

12 syllables

$$
10
$$

3

$77 \%$

5

abba (II)
alternance de : $\mathrm{MF}$

II,e, o,y

В,Р, ч, З, Н, Нь
Remarques

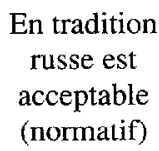

estimation approximative Mallarmé: 13 pauses Volochine: 10 pauses est accept. en tradition russe (normatif) il s'agit seulement des effets des sons 


\section{NOTES}

1. Pour le temps de création du sonnet, ainsi que son analyse voir : Charles Chadwick (1962): Mallarmé, sa pensée dans la poésie, chap. 1, «La tentation du lyrisme», Paris, Librairie José Corti ; E. Noulet (1948): Dix poèmes de Stéphane Mallarmé, Genève; Guy Michaud (1953) : Mallarmé, l'homme et l'auvre, Paris, HatierBoivin.

2. A. Bauler (1905) : «Stéphane Mallarmé», Voprosy zhizni, 2 (Feb.-Mar.), St. Petersburg, pp. $61-100$.

3. V. Brussov (1907): «Lebed’. Sonet Stefana Mallarme», Khrizopras, Iz-vo «Samotsvet», p. 5. Reprint by Graphic and Printing Institute, Tel Aviv, 1972

4. R. Ghil (1908): «Pis'ma o frantsuzskoi pœzii II, Stefan Mallarme. Pervye Proizvedenia», Vesy, 8, p. 108.

5. Ibid., p. 107.

6. Volochine (1978) : «Pis'mo k materi ot 7 janv. 1914 g», tsit. Kuprianovym, Sud'ba pota, Kiev, Naukova Dumka, p. 124

7. Volochine (1910) : «Anri de Ren'e», Apollon, 4, p. 89.

8. Volochine: «Pis'mo k materi ot 7 janv. 1914 g», p. 124

9. Voir I. Kuprianov (1978) : Sud' ba pceta, Kiev, Naukova Dumka.

10. N. Achukin (1929): Valerii Brussov, Moskva, p. 347. Brussov a oublié Viacheslav Ivanov qui a fait des beaux sonnets aussi.

11. Volochine (1909) : «Horomedon», Zoloto Runo, 11-12, p. 55.

12. Ibid, p. 59 .

13. Ibid., p. 57

14. S. Mallarmé (1941): «Salut», Poésie, Éd. complète, Paris, N.R.F., p. 18.

15. Volochine (1918) : Iverni (Izbrannye Proizvedenia), Moskva, p. 3.

16. Volochine (1907): «Émile Verhaeren et Valéry Brussov», Vesy, 2, p. 76.

17. Volochine, «Pis'mo k M.V. Sabachnikovu», hiver 1912-1913, cité par V. Perelmouter dans: «Tretil sobesednik», Masterstvo Perevoda, 12, 1981, p. 421.

18. Volochine : «Émile Verhaeren et Valéry Brussov», p. 75.

19. Volochine (1919) : «Predvarenie o perevodakh», Verhaeren. Sud" ba. Tvorchestvo. Perevody, Moskva, Tvorchestvo, p. 29.

20. De la conversation de Gauthier avec Émile Berger, citée par Volochine (1908) dans «Liki tvorchestva. Pol Verlen», Izdatel'stvo «Fakely», pp. 191-192.

21. Ibid., p. 197.

22. Ibid., p. 198.

23. R. Jakobson (1963): «Aspects linguistiques de la traduction», Essais de linguistique générale, Paris, Édition de Minuit, p. 86.

24. L. Robel (1968) : «Problèmes théoriques de la traduction de la poésie russe en français», Revue des études slaves, 47, p. 128.

25. E. Rice (1982): «Maximillian Volochine i ego vremia», Maximillian Volochine. Stikhotvorenia, vol, I, Paris, YMCA Press, p. xlv.

26. Ch. Chassé (1954) : Les Clefs de Mallarmé, Paris, Aubier, Éd. Montaigne, pp. 152-153.

27. Le rétrécissement est la technique par laquelle l'image similaire est transmise avec un sens plus faible (terminologie - V.A.).

28. Volochine: «Horomedon», p. 60.

29. Chadwick note que Mallarmé emploie «se délivrer» dans la connotation anglaise du mot. Voir Chadwick, p. 26.

30. Pour les symboles de Mallarmé, voir E. Noulet: op. cit., p. 613.

31. J. Gengoux (1950) : Le Symbolisme de Mallarmé, Paris, Nizet, p. 613.

32. Cité par G. Michaud, p. 120.

33. Ch. Chadwick, p. 20.

34. K. Gan'china (1962) : Frantsusko-russkii slovar', Moskva, p. 408.

35. C.f. Gengoux, p. 139.

36. C. Mauron (1968): Mallarmé, l'obscur, Paris, p. 153

37. Pour la synesthésie, chez les symbolistes français, voir Svend Johansen (1945): Etude sur le style des symbolistes français, Copenhague, Einar Munksgaard, p. 18.

38. Chadwick, p. 20.

39. C.f. V. Gofman (1937) : «Jazyk symvolistov», Literatournœ Nasledstvo, 27-28, p. 82.

40. L. Robel, p. 129 .

41. Volochine : «Predvarenie o perevodakh», p. 29. 


\section{Le cygne}

1 Le vierge, le vivace et le bel aujourd'hui

2 Va-t-il nous déchirer avec un coup d'aile ivre

3 Ce lac dur oublié que hante sous le givre

4 Le transparent glacier des vols qui n'ont pas fui!

5 Un cygne d'autrefois se souvient que c'est lui

6 Magnifique mais qui sans espoir se délivre

7 Pour n'avoir pas chanté la région où vivre

8 Quand du stérile hiver a resplendi l'ennui.

9 Tout son col secouera cette blanche agonie

10 Par l'espace infligée à l'oiseau qui le nie,

11 Mais non l'horreur du sol où le plumage est pris

12 Fantôme qu'à ce lieu son pur éclat assigne,

13 Il s'immobilise au songe froid de mépris

14 Que vêt parmi l'exil inutile le Cygne.

\section{ЛЕБЕДЬ}

1 Могучий, девственный, в красе извивных линий

2 Безумием крыла ужель не разорвет

3 Он озеро мечты, где скрыл узорный иней

4 Полетов скованных прозрачно-синий лед.

5 И лебедь прежнихъ дней, в порыве гордой муки,

6 Он знает, что ему не взвиться, не запеть:

7 Не создал в песне он страны, чтоб улететь,

8 Когда придет зима в сиянье белой скуки.

9 Он шеей отряхнет смертельное бессилье,

10 Которым вольного теперь неволит даль,

11 Но не позор земли, что приморозил крылья.

12 Он скован белизной земного одеянья

13 И стынет в гордых снах ненужного изгнанья,

14 Окутанный в надменную печаль. 